\title{
FAITHFUL, IRREDUCIBLE $*$-REPRESENTATIONS FOR GROUP ALGEBRAS OF FREE PRODUCTS
}

\author{
by M. J. CRABB and C. M. MCGREGOR
}

(Received 23rd October 1997)

\begin{abstract}
Let $G$ be the free product of groups $A$ and $B$, where $|A| \geq 3$ and $|B| \geq 2$. We construct faithful, irreducible *-representations for the group algebras $\mathbb{C}[G]$ and $\ell^{\prime}(G)$. The construction gives a faithful, irreducible representation for $F[G]$ when the field $\mathbf{F}$ does not have characteristic 2 .
\end{abstract}

1991 Mathematics subject classification: 22D10, 22D20.

\section{Introduction}

Let $G$ be the free product of groups $A$ and $B$, where $|A| \geq 3$ and $|B| \geq 2$. Let $\mathbb{F}$ be any field and let $\mathbb{C}$ be the complex field. Denote by $\mathbb{F}[G]$ the group algebra over $\mathbb{F}$ consisting of the finite sums of the form $\sum_{1}^{n} \alpha_{i} g_{i}\left(n \in \mathbb{N}, \alpha_{i} \in \mathbb{F}, g_{i} \in G\right)$, and denote by $\ell^{1}(G)$ the group algebra over $\mathbb{C}$ consisting of the infinite sums of the form $\sum_{1}^{\infty} \alpha_{i} g_{i}$ $\left(\alpha_{i} \in \mathbb{C}, g_{i} \in G, \sum_{1}^{\infty}\left|\alpha_{i}\right|<\infty\right)$.

The primitivity of $\mathbb{F}[G]$ was established by Formanek in [1] using comaximal ideals. In certain special cases, faithful, irreducible representations have been constructed: in [5] for $\mathbb{C}[G]$ and $\ell^{1}(G)$ where $G$ is the free group on a countable set with at least two elements; and by Irving in [2] and [3] for $\mathbb{F}[G]$ in the cases where $A$ is infinite and residually $<|B|$, and where $A$ and $B$ are finite with $B$ cyclic. A related topic was dealt with by Paschke and Salinas in [6] where it was shown that the $C^{*}$-algebra $C_{r}^{*}(G)$ generated by the regular representation of $G$ is simple and therefore primitive.

Here we construct faithful, irreducible $*$-representations $\pi$ of $\mathbb{C}[G]$ and $\ell^{1}(G)$ on inner product spaces $L$. In particular, this guarantees the primitivity of $\ell^{1}(G)$ which was not covered in [1]. The method also gives explicit faithful, irreducible representations of $\mathbb{F}[G]$ when $\mathbb{F}$ is any field not of characteristic 2 .

Our construction technique has two variations which we refer to as the character method and the identification method. The essential difference lies in the way they yield irreducibility. We use the first in Sections 2 and 3 to deal with $\mathbb{C}[G]$ in two cases when $d^{2}=1$ for all $d \in B$. Then $B$ is abelian and so admits a non-trivial homomorphism into $\mathbb{C}$, i.e. a character. The second method, which is applied in Sections 4 and 5 to the two remaining $\mathbb{C}[G]$ cases, involves identifying certain pairs of basis vectors from the earlier cases. The constructions for $\ell^{\prime}(G)$ are essentially the same as those for $\mathbb{C}[G]$ but the proofs require modification. These are dealt with in Section 6. 
For $g=d_{1} d_{2} \ldots d_{m} \in G \backslash\{1\}$, where $d_{i} \in A \backslash\{1\}$ ( $i$ odd) and $d_{i} \in B \backslash\{1\}$ (i even), or vice versa, define

$$
\beta(g)=d_{1}, \quad \varepsilon(g)=d_{m}, \quad \lambda(g)=m, \quad \beta(1)=\varepsilon(1)=1, \quad \lambda(1)=0
$$

We call $d_{1} d_{2} \ldots d_{m}$ the reduced form of $g$. A subset $H$ of $G$, containing 1 , is right closed if right segments of $g \in H$ are also in $H$, i.e. $g \in H$ with reduced form $d_{1} \ldots d_{m}$ implies $d_{k} \ldots d_{m} \in H(k=1, \ldots, m)$.

In each case we construct a subset $W$ of $G$ which acts as an orthonormal basis for $L$. So expressions of the form $\sum \alpha_{i} v_{i}$ where $\alpha_{i} \in \mathbb{C}$ and $v_{i} \in G$ may represent vectors in $L$ or elements of the algebra $\mathbb{C}[G]$ or $\ell^{\prime}(G)$. For $x=\sum \alpha_{i} w_{i}$ and $y=\sum \beta_{i} w_{i}$ in $L$, where $\alpha_{i}, \beta_{i} \in \mathbb{C}$ and $w_{i}$ are distinct in $W$, the inner product on $L$ is given by

$$
\langle x, y\rangle=\sum \alpha_{i} \bar{\beta}_{i}
$$

For $f=\sum \alpha_{i} g_{i}$ in $\mathbb{C}[G]$ or $\ell^{1}(G)$, where $\alpha_{i} \in \mathbb{C}$ and $g_{i} \in G$, define

$$
f^{*}=\sum \overline{\alpha_{i}} g_{i}^{-1}
$$

It is with respect to this involution that $\pi$ is a $*$-representation, i.e. for $x, y \in L$,

$$
\langle\pi(f) x, y\rangle=\left\langle x, \pi\left(f^{*}\right) y\right\rangle
$$

For a subset $X$ of the vector space $L$, write $-X$ for $\{-x: x \in X\}, \pm X$ for $X \cup(-X), \operatorname{lin}(X)$ for the linear span of $X$, and $\operatorname{supp}(X)$ for the support of $X$, i.e. the set of $w \in W$ such that, for some $x \in X,\langle x, w\rangle \neq 0$.

\section{Character method - first case}

Assume that $d^{2}=1$ for all $d \in B$ and fix $b \in B \backslash\{1\}$. Assume also that there exists $a \in A$ with $a^{2} \neq 1$. Fix this $a$ and let $c=b a$. Construct the inner product space $L$ consisting of sums of the form $\sum_{1}^{n} \alpha_{i} w_{i}\left(n \in \mathbb{N}, \alpha_{i} \in \mathbb{C}, w_{i} \in W\right)$ with orthonormal basis

$$
W=T \cup U_{A} \cup U_{B} \cup V_{A} \cup V_{B} \subseteq G
$$

where

$$
\begin{aligned}
T & =\left\{g\left(b a^{2}\right)^{n(n-1) / 2}: n \in \mathbb{N}, g \in G, \lambda(g) \leq 2 n, \varepsilon(g) \in A\right\}, \\
S & =\left\{g\left(b a^{2}\right)^{n(n-1) / 2}: n \in \mathbb{N}, g \in G, \lambda(g)=2 n, \varepsilon(g) \in A, g \neq\left(b a^{2}\right)^{n}\right\}, \\
U_{B} & =\left\{c^{k} v: v \in S, k \geq 0\right\}, \quad U_{A}=a U_{B}, \\
V_{A} & =\left\{a^{\prime} u: a^{\prime} \in A \backslash\{1, a\}, u \in U_{B}\right\}, \quad V_{B}=\left\{b^{\prime} u: b^{\prime} \in B \backslash\{1, b\}, u \in U_{A}\right\} .
\end{aligned}
$$


Observe that $1 \in T$ (take $n=1$ and $g=1$ ). Also, $\beta(w) \in B$ for all $w \in S$, and so for all $w \in U_{B}$. It follows that $\beta(w) \in A$ for $w \in U_{A} \cup V_{A}$ and $\beta(w) \in B$ for $w \in V_{B}$. The sets $T$, $U_{A}, U_{B}, V_{A}$ and $V_{B}$ are pairwise disjoint except that $T \cap U_{B}=S$. Also, $U_{B} \backslash S=b U_{A}$. Since $c \in S$ (take $n=1$ and $g=c$ ) it follows that $c^{k} \in U_{B} \backslash S$ for $k>1$. We further define $U_{B}^{*} \subseteq U_{B}$ and $V_{A}^{*} \subseteq V_{A}$ by

$$
U_{B}^{*}=\left\{c^{2^{n}}: n \in \mathbb{N}\right\} \text { and } V_{A}^{*}=a^{2} U_{B}^{*}
$$

\section{Lemma 2.1. The sets $W$ and $T$ are right closed.}

Proof. Let $w \in W$ have reduced form $d_{1} \ldots d_{m}$. It is enough to consider the right segment $w^{\prime}=d_{2} \ldots d_{m}$. This is straightforward for $w \in U_{A} \cup\left(U_{B} \backslash S\right) \cup V_{A} \cup V_{B}$ and for $w=g\left(b a^{2}\right)^{n(n-1) / 2} \in T$ with $\lambda(g)>0$. If $w=\left(b a^{2}\right)^{n(n-1) / 2}=\left(b a^{2}\right)^{n-1}\left(b a^{2}\right)^{(n-1)(n-2) / 2}$, where $n \geq 2$, then $w^{\prime}=g^{\prime}\left(b a^{2}\right)^{(n-1)(n-2) / 2}$, where $\lambda\left(g^{\prime}\right)=2 n-3$. Hence $w^{\prime} \in T$.

Lemma 2.2. Let $w \in W$. Then

$$
\begin{aligned}
& \text { either }(A \backslash\{1\}) w \subseteq W \text { or }(A \backslash\{1\}) w \subseteq G \backslash W, \text { and } \\
& \text { either }(B \backslash\{1\}) w \subseteq W \text { or }(B \backslash\{1\}) w \subseteq G \backslash W .
\end{aligned}
$$

Proof. Let $w=g\left(b a^{2}\right)^{n(n-1) / 2} \in T$. Without loss suppose that $g \neq\left(b a^{2}\right)^{n}$. For $d \in A \cup B,|\lambda(d g)-\lambda(g)| \leq 1$, and if $\lambda(d g)=2 n+1$ then $d w \in U_{A} \cup V_{A}$. Hence $d w \in W$ if $\lambda(g)>0$. If $\lambda(g)=0$ (i.e. $g=1)$ and $n>1$ then $(A \backslash\{1\}) w \subseteq T$, and writing $w=\left(b a^{2}\right)^{n-1}\left(b a^{2}\right)^{(n-1)(n-2) / 2}$ we see that $(B \backslash\{1\}) w \subseteq T$. If $g=1$ and $n=1$, then $w=1$, so that $(A \backslash\{1\}) 1=A \backslash\{1\} \subseteq T$ and $(B \backslash\{1\}) 1=B \backslash\{1\} \subseteq G \backslash W$.

Let $w \in U_{A} \cup V_{A}$, so that $w=a^{\prime} u$ with $a^{\prime} \in A \backslash\{1\}$ and $u \in U_{B}$. If $d \in A$ then $d w=$ $d a^{\prime} u \in U_{A} \cup V_{A} \cup U_{B}$ with the last case if $d^{-1}=a^{\prime}$. Similarly, $d w \in W$ if $w \in\left(U_{B} \backslash S\right) \cup V_{B}$ and $d \in B$.

If $w \in U_{A}$ and $d \in B \backslash\{1\}$ then $d w \in U_{B} \cup V_{B}$. If $w \in U_{B}$ and $d \in A \backslash\{1\}$ then $d w \in U_{A} \cup V_{A}$.

Let $w \in V_{A}$ and $d \in B \backslash\{1\}$. So $w=a^{\prime} u$ with $a^{\prime} \in A \backslash\{1, a\}$ and $u \in U_{B}$. Now $d w=$ $d a^{\prime} u \notin T$ since its right segment $a^{\prime} u \in V_{A}$. Since $a^{\prime} u \notin U_{A}, d w \notin U_{B} \cup V_{B}$. Since $\beta(d w) \in B$, $d w \notin U_{A} \cup V_{A}$. Hence $d w \notin W$.

Similarly, if $w \in V_{B}$ and $d \in A \backslash\{1\}$ then $d w \notin W$.

Since $d^{2}=1$ for all $d \in B$, we can fix a homomorphism $\chi: B \rightarrow\{-1,1\} \subseteq \mathbb{C}$ with $\chi(b)=-1$. For $d \in A \cup B$, let $\pi(d): L \rightarrow L$ be the linear mapping defined, for $w \in W$, by

$$
\pi(d) w= \begin{cases}d w & \text { if } d w \in W \\ \chi(d) w & \text { if } w \in V_{A}^{*} \text { and } d \in B \backslash\{1\} \\ w & \text { in other cases when } d w \notin W\end{cases}
$$


Note that $\pi(1)$ is the identity operator on $L$. For $w \in V_{A}^{*}$ and $d_{1}, d_{2} \in B$ we have

$$
\pi\left(d_{1}\right) \pi\left(d_{2}\right) w=\pi\left(d_{1}\right) \chi\left(d_{2}\right) w=\chi\left(d_{1}\right) \chi\left(d_{2}\right) w=\chi\left(d_{1} d_{2}\right) w=\pi\left(d_{1} d_{2}\right) w .
$$

It is straightforward to verify that $\pi\left(d_{1}\right) \pi\left(d_{2}\right)=\pi\left(d_{1} d_{2}\right)$ if $d_{1}$ and $d_{2}$ are both in $A$ or both in $B$. So we may extend $\pi$ to $G$ and thence to a representation of $\mathbb{C}[G]$.

Let $w \in W$ with $w=d_{m} \ldots d_{2} d_{1}$ in reduced form. Since $d_{1} \in W, \pi\left(d_{1}\right) 1=d_{1}$ and, similarly, $\pi\left(d_{2} d_{1}\right) 1=d_{2} d_{1}$. Continuing, it follows that $\pi(w) 1=w$. Also,

$$
\pi\left(w^{-1}\right) w=\pi\left(w^{-1}\right) \pi(w) 1=\pi(1) 1=1 \quad(w \in W) .
$$

From the proof of Lemma 2.2 we have, for $d \in A \cup B$ and $w \in W$,

$$
\pi(d) w= \begin{cases}d w & \text { if } d w \in W, \\ w & \text { if } d \in A \text { and } w \in V_{B}, \\ w & \text { if } d \in B \text { and } w \in\left(V_{A} \backslash V_{A}^{*}\right) \cup\{1\}, \\ \chi(d) w & \text { if } d \in B \text { and } w \in V_{A}^{*},\end{cases}
$$

which separates the cases when $d w \notin W$.

For $d \in A \cup B, \pi(d) \pi\left(d^{-1}\right)=\pi(1)$ and $\pi(d)$ acts on $W$ as a permutation followed by scalar multiplication by \pm 1 . Therefore $\pi(d)$ is a unitary operator on $L, \pi\left(d^{-1}\right)=\pi(d)^{*}$ and $\pi$ is a $*$-representation of $\mathbb{C}[G]$.

Proposition 2.3. The representation $\pi$ is faithful.

Proof. Let $f \in \mathbb{C}[G] \backslash\{0\}$. Then $f=\sum_{1}^{n} \alpha_{i} g_{i}$ where $\alpha_{i} \in \mathbb{C}$ and $g_{i} \in G$. Choose an integer $k>\max \left\{\lambda\left(g_{i}\right): i=1, \ldots, n\right\}$. Consider $w=g\left(b a^{2}\right)^{k(k-1) / 2} \in T$ where $g \in G$ is such that $\lambda(g)=k$ and $\varepsilon(g) \in A$. For $i=1, \ldots, n$, we have $0<\lambda\left(g_{i} g\right)<2 k$ and $\varepsilon\left(g_{i} g\right)=\varepsilon(g)$. So $g_{i} w \in T$ and each right segment of $g_{i} w$ is in $T$. Hence $\pi\left(g_{i}\right) w=g_{i} w$ and $\pi(f) w=f w \neq 0$ since $f w w^{-1}=f \neq 0$. Thus $\pi(f) \neq 0$.

Notes 2.4. (1) With the notation of the above proof, if $g_{i}$ are distinct in $G$ then $g_{i} w$ are distinct in $T$. This will be used in the proof of Proposition 6.1.

(2) In the proof, the restrictions imposed on $A$ and $B$ at the beginning of Section 2 are not invoked. Hence, the proposition holds for the cases in Sections 3, 4 and 5. The same is true of the next lemma.

Lemma 2.5. (i) Let $w \in U_{B}$. Then $\pi(c) w \in U_{B} \backslash S$.

(ii) Let $w \in T$. Then $\pi\left(c^{n}\right) w \in U_{B}$ for all sufficiently large $n$.

Proof. (i) This follows directly from the definitions which give $\pi(c) w=c w$.

(ii) Write $w=g\left(b a^{2}\right)^{m(m-1) / 2}$ with $\lambda(g) \leq 2 m, \varepsilon(g) \in A$ and $g \neq\left(b a^{2}\right)^{m}$.

(a) Let $\lambda(g)=2 m$. Then $w \in S$ and $\pi\left(c^{n}\right) w=c^{n} w \in U_{B}$ for all $n \in \mathbb{N}$.

(b) Let $\lambda(g)$ be even and $<2 m$. Then $\beta(g) \in B$, and $k=m-\frac{1}{2} \lambda(g)$ gives $\pi\left(c^{k}\right) w=$ 
$c^{k} w \in S$ and $\pi\left(c^{n}\right) w \in U_{B}$ for all $n>k$, by (a).

(c) Now suppose that $\lambda(g)$ is odd. Then $\beta(g) \in A$ and we can write $w=c^{-p} w^{\prime}$ where $p \geq 0, \beta\left(w^{\prime}\right) \in A$ and $w^{\prime} \neq c^{-1} g^{\prime}$ with $g^{\prime} \in G$ and $\beta\left(g^{\prime}\right) \in A$. Since each right segment of $w$ is in $T, \pi\left(c^{p}\right) w=c^{p} w=w^{\prime}$ and $w^{\prime} \in T$. Hence we can reduce to the case $w \in T$, $\beta(w) \in A$ and $w \neq c^{-1} g^{\prime}$ with $g^{\prime} \in G$ and $\beta\left(g^{\prime}\right) \in A$. If $\lambda(w) \geq 3, c$ does not cancel completely into $w$ and so $\beta(c w) \in B$. Since $\lambda(c w) \leq \lambda(w)+1, c w \in T$ and so $\pi(c) w=c w$. Now cases (a) and (b) give $\pi\left(c^{n}\right) w \in U_{B}$ for all sufficiently large $n$.

Finally, if $\lambda(w)=1$ then $w=a^{\prime} \in A \backslash\{1\}$. If $a^{\prime} \neq a^{-1}$ then $\pi(a) w=a a^{\prime}$ and $\pi(c) w=b a a^{\prime} \in T$ which gives $\pi\left(c^{n}\right) w \in U_{B}$ for all sufficiently large $n$, by (a) and (b). Also, $\pi(a) a^{-1}=1$ and $\pi(c) a^{-1}=\pi(b) 1=1$. Then $\pi\left(c^{r}\right) 1=c^{r} \in U_{B}$ for all $r \in \mathbb{N}$ which gives the result in the last case.

We now define

$$
\begin{aligned}
V_{A}^{0} & =\left\{a^{-s} u: s \in \mathbb{N}, a^{-s} \notin\{1, a\}, u \in U_{B}\right\}, \\
V_{A}^{\infty} & =\left\{a^{\prime} u: a^{\prime} \in A \backslash\left\{a^{t}:-\infty<t \leq 1\right\}, u \in U_{B}\right\} .
\end{aligned}
$$

Thus $V_{A}=V_{A}^{0} \cup V_{A}^{\infty}$ and $V_{A}^{0} \cap V_{A}^{\infty}=\emptyset$. Note that $V_{A}^{\infty}$ may be empty.

Lemma 2.6. For $d \in\{a, b\}$,

$$
\pi(d) V_{A}^{\infty} \subseteq \pm V_{A}^{\infty} \text { and } \pi(d) V_{B} \subseteq V_{B}
$$

Proof. Consider $w \in V_{A}^{\infty}$ so that $w=a^{\prime} u$ where $a^{\prime} \in A \backslash\left\{a^{t}:-\infty<t \leq 1\right\}$ and $u \in U_{B}$. Then $\pi(a) w=a a^{\prime} u$ and $a a^{\prime} \in A \backslash\left\{a^{t}:-\infty<t \leq 1\right\}$. Hence $a a^{\prime} u \in V_{A}^{\infty}$. Also, $\pi(b) w= \pm w \in \pm V_{A}^{\infty}$.

The case of $V_{B}$ follows directly from the definitions together with the assumption that $b^{2}=1$ which guarantees that, for $b^{\prime} \in B \backslash\{b\}, b b^{\prime} \neq 1$.

Lemma 2.7. Let $w \in U_{A} \cup V_{A}^{0}$. Then $\pi\left(c^{n}\right) w \in \pm\left(U_{B} \cup V_{A}^{\infty}\right)$ for all sufficiently large $n$.

Proof. (i) Assume $a$ has finite period $p$. Let $w \in U_{A} \cup V_{A}^{0}$. Write $w=a^{r} u$ with $1 \leq r<p$ and $u \in U_{B}$. Then $\pi(c) w=\pi(b) a^{r+1} u= \pm a^{r+1} u$ if $a^{r+1} \neq 1$. Continuing, we have

$$
\pi\left(c^{p-r}\right) w= \pm \pi(b) a^{p} u= \pm \pi(b) u= \pm b u
$$

Now, if $u \in S$ then $b u \in T$ and Lemma 2.5 gives $\pi\left(c^{n}\right) w \in \pm U_{B}$ for all sufficiently large $n$. If $u \in U_{B} \backslash S$ then $u=c^{k} v$ with $k \in \mathbb{N}$ and $v \in S$. Hence, when $w=a^{r} c^{k} v$, we have $\pi\left(c^{p-r}\right) w= \pm b u= \pm a c^{k-1} v$. Continuing, we have, for some $m \in \mathbb{N}$, that $\pi\left(c^{m}\right) w= \pm a v$. Then the first part gives $\pi\left(c^{n}\right) w \in \pm U_{B}$ for all sufficiently large $n$.

(ii) Assume $a$ has infinite period. If $w=a u \in U_{A}$, where $u \in U_{B}$, then 


$$
\pi(c) w=\pi(b) a^{2} u= \pm a^{2} u \in \pm V_{A}^{\infty}
$$

and so $\pi\left(c^{n}\right) w \in \pm V_{A}^{\infty}$ for all $n \in \mathbb{N}$, by Lemma 2.6. If $w=a^{-s} u \in V_{A}^{0}$, where $s \in \mathbb{N}$ and $u \in U_{B}$, then $\pi(c) w=\pi(b) a^{1-s} u=a^{1-s} u$ if $s>1$. Continuing, we have $\pi\left(c^{s}\right) w=\pi(b) u=$ $b u$. If $u \in S$ then $b u \in T$, and if $u \in U_{B} \backslash S$ then $b u \in U_{A}$. Hence, the above gives $\pi\left(c^{n}\right) w \in \pm\left(U_{B} \cup V_{A}^{\infty}\right)$ for all sufficiently large $n$.

Proposition 2.8. Let $X$ be a finite subset of $W$ and let $x_{0} \in X$. Then there exists $h \in \mathbb{C}[G]$ such that $\pi(h) x_{0}=1$ and $\pi(h) x=0$ for all $x \in X \backslash\left\{x_{0}\right\}$.

Proof. Consider first the case $X \subseteq U_{B} \cup V_{A}^{\infty}$ and $x_{0}=c^{m_{1}} \in X$. Write the distinct elements of $X \cap U_{B}$ as $c^{m_{1}}, \ldots, c^{m_{x}}, c^{n_{1}} v_{1}, \ldots, c^{n_{\beta}} v_{\beta}$, where $m_{i} \in \mathbb{N}, n_{j} \in \mathbb{N} \cup\{0\}$ and $v_{j} \in S \backslash\{c\}$. Consider $X_{1}=\pi\left(c^{p}\right) X$, where $p \in \mathbb{N}$. Since $\pi(c) V_{A}^{\infty} \subseteq \pm V_{A}^{\infty}$ and $\pi(c) U_{B} \subseteq U_{B}$, we have $X_{1} \subseteq U_{B} \cup\left( \pm V_{A}^{\infty}\right)$ and

$$
X_{1} \cap U_{B}=\left\{c^{p+m_{1}}, \ldots, c^{p+m_{x}}, c^{p+n_{1}} v_{1}, \ldots, c^{p+n_{\beta}} v_{\beta}\right\}
$$

For each $j, c^{p+n_{j}} v_{j} \neq c^{k}$ for any $k$ and so $c^{p+n_{j}} v_{j} \notin U_{B}^{*}$. Choose $p$ such that $p+m_{1}$ is the only power of 2 among the $p+m_{i}$. Then $X_{1} \cap U_{B}^{*}=\left\{c^{p+m_{1}}\right\}=X_{1} \cap\left( \pm U_{B}^{*}\right)$. Put $X_{2}=$ $\pi\left(a^{2}\right) X_{1}=\pi\left(a^{2} c^{p}\right) X$. Then $X_{2} \subseteq \pm V_{A}$ since $\pi\left(a^{2}\right) U_{B} \subseteq V_{A}$ and $\pi(a) V_{A}^{\infty} \subseteq V_{A}^{\infty}$. Also $x_{0}=c^{m_{1}} \in X$ gives $a^{2} c^{p} x_{0} \in X_{2} \cap V_{A}^{*}$. If $x \in X$ and $\pi\left(a^{2} c^{p}\right) x \in \pm V_{A}^{*}$ then $\pi\left(a^{-2}\right) \pi\left(a^{2} c^{p}\right) x=$ $\pi\left(c^{p}\right) x \in X_{1} \cap\left( \pm U_{B}^{*}\right)$. Hence $\pi\left(c^{p}\right) x=c^{p} x_{0}=\pi\left(c^{p}\right) x_{0}$ which gives $x=x_{0}$. So, if $x \in X \backslash\left\{x_{0}\right\}$ then $\pi\left(a^{2} c^{p}\right) x \in \pm\left(V_{A} \backslash V_{A}^{*}\right)$, and hence, by $(2.2), \pi(1-b) \pi\left(a^{2} c^{p}\right) x=0$. Also

$$
\pi\left((1-b) a^{2} c^{p}\right) x_{0}=\pi(1-b) a^{2} c^{p} x_{0}=2 a^{2} c^{p} x_{0}=2 a^{2} c^{p+m_{1}},
$$

since $a^{2} c^{p} x_{0} \in V_{A}^{*}$. Hence, if we take $h=\frac{1}{2}\left(a^{2} c^{p+m_{1}}\right)^{-1}(1-b) a^{2} c^{p}$, we have $\pi(h) x_{0}=1$ and $\pi(h) x=0$ for all $x \in X \backslash\left\{x_{0}\right\}$.

Now consider any finite $X \subseteq W$ and any $x_{0} \in X$. By replacing $X$ with $\pi\left(x_{0}^{-1}\right) X$ we may assume, by (2.1), that $x_{0}=1$. By Lemmas 2.5, 2.6 and 2.7 we have, for some $p \in \mathbb{N}$

$$
X_{3}=\pi\left(c^{p}\right) X \subseteq \pm\left(U_{B} \cup V_{A}^{\infty} \cup V_{B}\right)
$$

Then

$$
X_{4}=\pi(1-a) X_{3} \subseteq \operatorname{lin}\left(U_{A} \cup U_{B} \cup V_{A}^{\infty}\right)
$$

since $\pi(a) U_{B}=U_{A}$ and $\pi(1-a) V_{B}=\{0\}$. By Lemmas 2.6 and 2.7, for some $q \in \mathbb{N}$,

$$
X_{S}=\pi\left(c^{q}\right) X_{4} \subseteq \operatorname{lin}\left(U_{B} \cup V_{A}^{\infty}\right) .
$$

So, putting $h_{1}=c^{q}(1-a) c^{p}$, we have 


$$
X_{S}=\pi\left(h_{1}\right) X=\left\{\pi\left(c^{q+p}\right) x-\pi\left(c^{q} a c^{p}\right) x: x \in X\right\} .
$$

Suppose that $c^{q+p} \in \operatorname{supp}\left(X_{5}\right)$. Then, for some $x \in X, \pi\left(c^{q+p}\right) x= \pm c^{q+p}$ or $\pi\left(c^{q} a c^{p}\right) x=$ $\pm c^{q+p}$. In the first case, applying $\pi\left(c^{-q-p}\right)$ gives $x=1$. In the second case, applying $\pi\left(a^{-1} c^{-p}\right)$ gives $\pi\left(c^{q}\right) x= \pm a^{-1} c^{p} \in X_{3} \cap\left( \pm V_{A}^{0}\right)$, which is impossible. Thus $x_{1}=c^{q: p}$ appears in $\operatorname{supp}\left(X_{5}\right)$ only as $\pi\left(c^{q+p}\right) 1$. The first part now gives $h_{2} \in \mathbb{C}[G]$ such that $\pi\left(h_{2}\right) x_{1}=1$ and $\pi\left(h_{2}\right) x=0$ for all $x \in \operatorname{supp}\left(X_{5}\right) \backslash\left\{x_{1}\right\}$. Also, $\pi\left(h_{1}\right) 1=x_{1} \pm x_{2}$ with $x_{2} \in \operatorname{supp}\left(X_{5}\right) \backslash\left\{x_{1}\right\}$ and $\pi\left(h_{1}\right) x \in \operatorname{lin}\left(\operatorname{supp}\left(X_{5}\right) \backslash\left\{x_{1}\right\}\right)$ if $x \in X \backslash\{1\}$. Hence $\pi\left(h_{2} h_{1}\right) 1=1$ and $\pi\left(h_{2} h_{1}\right) x=0$ for all $x \in X \backslash\{1\}$. So $h=h_{2} h_{1}$ has the desired effect.

Corollary 2.9. The representation $\pi$ is irreducible.

Proof. Let $x=\sum_{1}^{n} \alpha_{i} w_{i} \in L$ with $\alpha_{i} \in \mathbb{C}, \alpha_{1} \neq 0$ and $w_{i}$ distinct in $W$. By Proposition 2.8 , there exists $h \in \mathbb{C}[G]$ such that $\pi(h) w_{1}=1$ and $\pi(h) w_{i}=0$ for $i \in\{2, \ldots, n\}$. Hence $\pi(h) x=\alpha_{1} 1$. Since $\pi(w) 1=w$ for all $w \in W, 1$ in $L$ is cyclic. Hence $x$ is cyclic and it follows that $\pi$ is irreducible.

\section{Character method - second case}

Assume that $d^{2}=1$ for all $d \in A$ and all $d \in B$. Fix $a_{1}, a_{2} \in A \backslash\{1\}$ with $a_{1} \neq a_{2}$, and fix $b \in B \backslash\{1\}$. Let $a_{3}=a_{1} a_{2}$ and observe that if $\{i, j, k\}=\{1,2,3\}$ then $a_{i} a_{j}=a_{k}$. Modify the construction of $W$ in Section 2 as follows. In the definitions of $T, S, U_{A}, U_{B}, V_{A}$, $V_{B}, U_{B}^{*}$ and $V_{A}^{*}$, where $a^{2}$ appears replace it with $a_{2}$, replace the other occurrences of $a$ with $a_{1}$, and take $c$ to be $b a_{1}$. Thus, in this case, $U_{A}=a_{1} U_{B}$ and $V_{A}^{*}=a_{2} U_{B}^{*}$. For $i=2,3$, let $V_{A}^{i}=a_{i} U_{B}$ and $V_{A}^{\prime}=V_{A} \backslash\left(V_{A}^{2} \cup V_{A}^{3}\right)$. Define $\pi$ as in Section 2. Then, arguing as before, we have that $\pi$ is a $*$-representation of $\mathbb{C}[G]$.

Proposition 3.1. The representation $\pi$ is faithful.

Proof. Follow the proof of Proposition 2.3, replacing $a^{2}$ with $a_{2}$.

Lemma 3.2. Let $w \in T \cup U_{A}$. Then $\pi\left(c^{n}\right) w \in U_{B}$ for all sufficiently large $n$.

Proof. For $w \in T$, the proof follows that of Lemma 2.5.

Let $w=a_{1} c^{k} v \in U_{A}$ where $k \geq 0$ and $v \in S$. If $k>0$ then $\pi(c) w=a_{1} c^{k-1} v$, which gives $\pi\left(c^{k}\right) w=a_{1} v$ and $\pi\left(c^{k+1}\right) w=b v \in T$. Apply the first part.

Lemma 3.3. For $d \in\left\{a_{1}, b\right\}$ and $w \in V_{A}$,

$$
\pi(d) V_{A} \subseteq \pm V_{A}, \quad \pi(d) V_{B} \subseteq V_{B}, \quad \pi\left(c^{2}\right) w= \pm w
$$

Also, 


$$
\pi(c) V_{A}^{2} \subseteq V_{A}^{3}, \quad \pi(c) V_{A}^{3} \subseteq \pm V_{A}^{2}, \quad \pi(c) V_{A}^{\prime} \subseteq V_{A}^{\prime}
$$

Proof. These follow directly from the definitions.

Proposition 3.4. Let $X$ be a finite subset of $W$ and let $x_{0} \in X$. Then there exists $h \in \mathbb{C}[G]$ such that $\pi(h) x_{0}=1$ and $\pi(h) x=0$ for all $x \in X \backslash\left\{x_{0}\right\}$.

Proof. Without loss, take $x_{0}=1$. By Lemmas 3.2 and 3.3 , for some $p \in \mathbb{N}$,

$$
X_{1}=\pi\left(c^{p}\right) X \subseteq \pm\left(U_{B} \cup V_{A} \cup V_{B}\right)
$$

As in Proposition 2.8, choose $p$ such that $\operatorname{supp}\left(X_{1}\right) \cap U_{B}^{*}=\left\{c^{\mathcal{P}}\right\}$. Then

$$
X_{2}=\pi\left(a_{2}\right) X_{1} \subseteq \pm\left(U_{A} \cup U_{B} \cup V_{A}^{2} \cup V_{A}^{\prime} \cup V_{B}\right)
$$

In view of Lemmas 3.2 and 3.3, again as in Proposition 2.8 we can choose $q$ sufficiently large, and here odd, such that

$$
X_{3}=\pi\left(c^{q}\right) X_{2} \subseteq \pm\left(U_{B} \cup V_{A}^{3} \cup V_{A}^{\prime} \cup V_{B}\right)
$$

and such that $\operatorname{supp}\left(X_{3}\right) \cap U_{B}^{*}=\emptyset$. Then

$$
X_{4}=\pi\left(a_{2}\right) X_{3} \subseteq \pm\left(U_{A} \cup V_{A}^{2} \cup V_{A}^{\prime} \cup V_{B}\right)
$$

and

$$
X_{5}=\pi(1-b) X_{4} \subseteq \operatorname{lin}\left(U_{A} \cup U_{B} \cup V_{B}\right),
$$

since $\operatorname{supp}\left(X_{4}\right) \cap V_{A}^{*}=\emptyset$. Then

$$
X_{6}=\pi\left(a_{3}\right) X_{5} \subseteq \operatorname{lin}\left(V_{A}^{2} \cup V_{A}^{3} \cup V_{B}\right)
$$

Suppose $n \in \mathbb{N}$ and $a_{2} c^{n} \in \operatorname{supp}\left(X_{6}\right)$. Then one of $\pi\left(a_{3}\right) a_{2} c^{n}=a_{1} c^{n}$ and $\pi\left(b a_{3}\right) a_{2} c^{n}=$ $c^{n+1}$ is in $\operatorname{supp}\left(X_{4}\right)$. Since $\operatorname{supp}\left(X_{4}\right) \cap U_{B}=\emptyset$, we have $a_{1} c^{n} \in \operatorname{supp}\left(X_{4}\right)$. Hence $\pi\left(a_{2} c^{-q} a_{2}\right) a_{1} c^{n} \in \pm \operatorname{supp}\left(X_{1}\right)$, i.e. $c^{n} \in \operatorname{supp}\left(X_{1}\right)$. For $m \in \mathbb{N}$, if $c^{m} \in X_{1}$ then its image in $X_{6}$ is $\pm a_{2} c^{m} \mp a_{3} c^{m+1}$. Hence

$$
c^{m} \in \operatorname{supp}\left(X_{1}\right) \Leftrightarrow a_{2} c^{m} \in \operatorname{supp}\left(X_{6}\right)
$$

Since $\operatorname{supp}\left(X_{1}\right) \cap U_{B}^{*}=\left\{c^{p}\right\}$, we have $\operatorname{supp}\left(X_{6}\right) \cap V_{A}^{*}=\left\{a_{2} c^{p}\right\}$, and $a_{2} c^{p}$ in $\operatorname{supp}\left(X_{6}\right)$ appears only in the image of 1 in $X$. Let $v=a_{2} c^{p}$. By Lemma 3.3,

$$
\begin{aligned}
& X_{7}=\pi(1-b) X_{6} \subseteq \operatorname{lin}\left(\{v\} \cup V_{B}\right) \\
& X_{8}=\pi\left(1-a_{1}\right) X_{7} \subseteq \operatorname{lin}\left(\left\{v-a_{1} v\right\}\right)
\end{aligned}
$$


It is straightforward to verify that 1 in $X$ maps to $v-a_{3} c^{p+1}$ in $X_{6}$, then to $2 v$ in $X_{7}$ and to $2\left(v-a_{1} v\right)$ in $X_{8}$. Other elements of $X$ map into $\operatorname{lin}\left(\left(V_{A} \backslash V_{A}^{*}\right) \cup V_{B}\right)$ in $X_{6}$, then to 0 in $X_{8}$. We have $\pi(1-b)\left(v-a_{1} v\right)=2 v$ since $a_{1} v=a_{3} c^{p} \in V_{A} \backslash V_{A}^{*}$. Hence

$$
h=\frac{1}{4} v^{-1}(1-b)\left(1-a_{1}\right)(1-b) a_{3}(1-b) a_{2} c^{q} a_{2} c^{p}
$$

gives $\pi(h) 1=1$ and $\pi(h) x=0$ for all $x \in X \backslash\{1\}$.

Corollary 3.5. The representation $\pi$ is irreducible.

Proof. Follow the proof of Corollary 2.9.

\section{Identification method - first case}

Assume there exists $a \in A$ with $a^{2} \neq 1$ and $b \in B$ with infinite period. Fix such $a$ and $b$ and let $c=b a$. Define sets $T, S, U_{A}, U_{B}, V_{A}, V_{B}$ and $U_{B}^{*}$ exactly as in Section 2. Define also

$$
V_{A B}=\left\{a^{2} u: u \in U_{B}\right\} \subseteq V_{A} \text { and } V_{B A}=\left\{b^{2} a u: u \in U_{B}\right\} \subseteq V_{B} .
$$

We identify each element of $V_{A B}$ with an element of $\pm V_{B A}$. Specifically, for $u \in U_{B}$, we identify $a^{2} u$ with $\lambda_{u} b^{2} a u$, and write

$$
a^{2} u \equiv \lambda_{u} b^{2} a u
$$

where $\lambda_{u} \in \mathbb{R}$ is given by

$$
\lambda_{u}=\left\{\begin{aligned}
1 & \text { if } u \in U_{B} \backslash U_{B}^{*}, \\
-1 & \text { if } u \in U_{B}^{*}
\end{aligned}\right.
$$

Thus, for all $u \in U_{B}, a^{2} u \equiv \pm b^{2} a u$. Here we take $L$ to be the inner product space with orthonormal basis

$$
W=T \cup U_{A} \cup U_{B} \cup\left(V_{A} \backslash V_{A B}\right) \cup\left(V_{B} \backslash V_{B A}\right) \cup V_{A B} .
$$

For $d \in A \cup B$, let $\pi(d): L \rightarrow L$ be the linear mapping defined, for $w \in W \backslash V_{A B}$, by

$$
\pi(d) w= \begin{cases}d w & \text { if } d w \in W, \\ w & \text { if } d w \notin W,\end{cases}
$$

and, for $w=a^{2} u \equiv \lambda_{u} b^{2} a u \in V_{A B}$ where $u \in U_{B}$, by

$$
\pi(d) w= \begin{cases}d a^{2} u & \text { if } d \in A, \\ \lambda_{u} d b^{2} a u & \text { if } d \in B\end{cases}
$$


Lemma 2.2 remains valid, so we may extend $\pi$ to $G$ and thence to a representation of $\mathbb{C}[G]$. As in Section 2, for $d \in A \cup B, \pi(d)$ is a unitary operator on $L$, and $\pi$ is a *representation.

Proposition 4.1. The representation $\pi$ is faithful.

Proof. Follow the proof of Proposition 2.3.

It follows, directly from the definitions, that

$$
\begin{aligned}
& \pi\left(1-a^{\prime}\right) w=0 \quad\left(a^{\prime} \in A, w \in V_{B} \backslash V_{B A}\right), \\
& \pi\left(1-b^{\prime}\right) w=0 \quad\left(b^{\prime} \in B, w \in V_{A} \backslash V_{A B}\right), \\
& \pi\left(a^{2}-b^{2} a\right) w= \begin{cases}0 & \text { if } w \in U_{B} \backslash U_{B}^{*}, \\
2 a^{2} w & \text { if } w \in U_{B}^{*} .\end{cases}
\end{aligned}
$$

Lemma 2.5 remains true since vectors in $V_{A B}$ do not appear in the calculation, and the proof is the same.

Define subsets of $V_{A}$ and $V_{B}$ as follows.

$$
\begin{aligned}
V_{A}^{0} & =\left\{a^{-s} u: s \in \mathbb{N}, a^{-s} \notin\left\{1, a, a^{2}\right\}, u \in U_{B}\right\}, \\
V_{A}^{\infty} & =\left\{a^{\prime} u: a^{\prime} \in A \backslash\left\{a^{s}:-\infty<s \leq 2\right\}, u \in U_{B}\right\}, \\
V_{B}^{0} & =\left\{b^{-s} u: s \in \mathbb{N}, u \in U_{A}\right\}, \\
V_{B}^{\infty} & =\left\{b^{\prime} u: b^{\prime} \in B \backslash\left\{b^{s}:-\infty<s \leq 2\right\}, u \in U_{A}\right\} .
\end{aligned}
$$

These four sets are disjoint. Their union is $\left(V_{A} \backslash V_{A B}\right) \cup\left(V_{B} \backslash V_{B A}\right)$. Note that $V_{A}^{0}$ and $V_{A}^{\infty}$ differ slightly from the corresponding sets in Section 2.

Lemma 4.2. For $d \in\{a, b\}$,

$$
\pi(d) V_{A}^{\infty} \subseteq V_{A}^{\infty} \text { and } \pi(d) V_{B}^{\infty} \subseteq V_{B}^{\infty}
$$

Proof. Consider $w=a^{\prime} u \in V_{A}^{\infty}$ where $a^{\prime} \in A \backslash\left\{a^{s}:-\infty<s \leq 2\right\}$ and $u \in U_{B}$. Then $\pi(a) w=a a^{\prime} u \in V_{A}^{\infty}$ since $a a^{\prime} \in A \backslash\left\{a^{s}:-\infty<s \leq 2\right\}$. Also, $\pi(b) w=w$. The case $w \in V_{B}^{\infty}$ is similar.

Lemma 4.3. (i) Let $w \in U_{A} \cup V_{B}^{0}$. Then $\pi\left(c^{n}\right) w \in \pm V_{B}^{\infty}$ for all sufficiently large $n$.

(ii) Let $w \in V_{A}^{0} \cup V_{A B}$. Then $\pi\left(c^{n}\right) w \in \pm\left(U_{B} \cup V_{A}^{\infty}\right)$ for all sufficiently large $n$.

Proof. (i) Consider $w=a u \in U_{A}$ where $u \in U_{B}$. Then 


$$
\pi(c) w=\pi(b) a^{2} u \equiv \pm \pi(b) b^{2} a u= \pm b^{3} a u \in \pm V_{B}^{\infty}
$$

since $b^{3} \in B \backslash\left\{b^{s}: s \leq 2\right\}$. Hence $\pi\left(c^{n}\right) w \in \pm V_{B}^{\infty}$ for all $n \in \mathbb{N}$.

Consider $w=b^{-s} u \in V_{B}^{0}$ where $s \in \mathbb{N}$ and $u \in U_{A}$. Then $\pi(a) w=w$ and $\pi(c) w=$ $\pi(b) w=b^{1-s} u$. Continuing, we get $\pi\left(c^{s}\right) w=u$. By the first part, $\pi\left(c^{n}\right) w \in \pm V_{B}^{\infty}$ for all $n \geq s+1$.

(ii) Consider $w=a^{-s} u$ where $s \in \mathbb{N}, u \in U_{B}$ and, if $a$ has finite period $p, s \leq p-2$. This includes any $w \in V_{A}^{0}$, together with $w \in V_{A B}$ in the case of $a$ having finite period.

Take first the case $u \in S$. If $s>1$ then $\pi(c) w=\pi(b) a^{1-s} u=a^{1-s} u$, and continuing we get $\pi\left(c^{s}\right) w=\pi(b) u=b u$, which also holds if $s=1$. Since $b u \in T$, this gives $\pi\left(c^{n}\right) w \in U_{B}$ for all sufficiently large $n$.

Now suppose that $u \in U_{B} \backslash S$, so that $w=a^{-s} u=a^{-s} c^{k} v$ where $k \in \mathbb{N}$ and $v \in S$. As above,

$$
\pi\left(c^{s}\right) w=b u=b^{2} a c^{k-1} v \equiv \pm a^{2} c^{k-1} v \in \pm V_{A B} .
$$

Now, $\pi(c) a^{2} c^{k-1} v=\pi(b) a^{3} c^{k-1} v$. If $a$ has infinite period then $\pi(b) a^{3} c^{k-1} v=a^{3} c^{k-1} v \in V_{A}^{\infty}$, and so $\pi\left(c^{n}\right) w \in \pm V_{A}^{\infty}$ for all $n>s$. If $a$ has finite period then $\pi\left(c^{3}\right) w$ is of the same form as $\pm w$, with $k-1$ in place of $k$. Repetition gives some $m \in \mathbb{N}$ such that $\pi\left(c^{m}\right) w= \pm a^{2} v$. Now the first part gives $\pi\left(c^{n}\right) w \in \pm U_{B}$ for all sufficiently large $n$.

Finally, if $a$ has infinite period and $w \in V_{A B}$ then $w=a^{2} u$ with $u \in U_{B}$. Hence, $\pi(c) w=a^{3} u \in V_{A}^{\infty}$ and $\pi\left(c^{n}\right) w \in V_{A}^{\infty}$ for all $n \in \mathbb{N}$.

Proposition 4.4. Let $X$ be a finite subset of $W$ and let $x_{0} \in X$. Then there exists $h \in \mathbb{C}[G]$ such that $\pi(h) x_{0}=1$ and $\pi(h) x=0$ for all $x \in X \backslash\left\{x_{0}\right\}$.

Proof. Without loss, take $x_{0}=1$. By Lemmas 4.2 and 4.3 , and results corresponding to Lemma 2.5 , for all sufficiently large $p \in \mathbb{N}$,

$$
X_{1}=\pi\left(c^{p}\right) X \subseteq \pm\left(U_{B} \cup V_{A}^{\infty} \cup V_{B}^{\infty}\right)
$$

The vector 1 in $X$ has image $c^{p}$ in $X_{1}$. As in Proposition 2.8, we choose $p$ such that $\operatorname{supp}\left(X_{1}\right) \cap U_{B}^{*}=\left\{c^{p}\right\}$. Let $h_{1}=\frac{1}{2}\left(a^{2}-b^{2} a\right) c^{p}$. Then $\pi\left(h_{1}\right) 1=a^{2} c^{p} \equiv-b^{2} a c^{p}$. Consider any $x \in X \backslash\{1\}$. Since $c^{p} \in \operatorname{supp}\left(X_{1}\right)$ can arise only from $1 \in X$, we have that $\pi\left(c^{p}\right) x \in$ $\pm\left(\left(U_{B} \backslash U_{B}^{*}\right) \cup V_{A}^{\infty} \cup V_{B}^{\infty}\right)$. Since $\pi\left(a^{2}-b^{2} a\right) w=0$ if $w \in U_{B} \backslash U_{B}^{*}$, and using Lemma 4.2, we deduce that $\pi\left(h_{1}\right) x \in \operatorname{lin}\left(V_{A}^{\infty} \cup V_{B}^{\infty}\right)$. Let $h_{2}=(1-a)(1-b)$. If $w \in V_{A}^{\infty}$ then $\pi(1-b) w=0$. If $w \in V_{B}^{\infty}$ then $\pi(1-b) w \in \operatorname{lin}\left(V_{B}^{\infty}\right)$ and $\pi\left(h_{2}\right) w=0$. Hence, $\pi\left(h_{2}\right) w=0$ if $w \in V_{A}^{\infty} \cup V_{B}^{\infty}$. Also, since $a^{2} c^{p} \equiv-b^{2} a c^{p}, \pi(b) a^{2} c^{p}=-b^{3} a c^{p}$ and hence

$$
\pi\left(h_{2}\right) a^{2} c^{p}=\pi(1-a)\left(a^{2} c^{p}+b^{3} a c^{p}\right)=a^{2} c^{p}-a^{3} c^{p}
$$

Let $h_{3}=h_{2} h_{1}$. Then, by above, $\pi\left(h_{3}\right) 1=a^{2} c^{p}-a^{3} c^{p}$ and $\pi\left(h_{3}\right) x=0$ for all $x \in X \backslash\{1\}$. Also, $\pi\left(a^{-2}\right)\left(a^{2} c^{p}-a^{3} c^{p}\right)=c^{p}-a c^{p}$. Next, 
and

$$
\pi\left(c^{-1}\right) a c^{p}=\pi\left(a^{-1} b^{-1}\right) a c^{p}=\pi\left(a^{-1}\right) b^{-1} a c^{p}=b^{-1} a c^{p} \in V_{B}^{0}
$$

$$
\pi\left(c^{-1}\right) b^{-1} a c^{p}=\pi\left(a^{-1} b^{-1}\right) b^{-1} a c^{p}=\pi\left(a^{-1}\right) b^{-2} a c^{p}=b^{-2} a c^{p} .
$$

Continuing, we get $\pi\left(c^{-p}\right) a c^{p}=b^{-p} a c^{p}$. Since $\pi\left(c^{-p}\right) c^{p}=1$, we have $\pi\left(c^{-p}\right)\left(c^{p}-a c^{p}\right)=$ $1-b^{-p} a c^{p}$. Then $\pi(1-a)\left(1-b^{-p} a c^{p}\right)=1-a$, and $\pi(b)(1-a)=1-c$ since $\pi(b) 1=1$. Further, since $a^{2} c \equiv b^{2} a c$ and $a^{2} c^{2} \equiv-b^{2} a c^{2}$,

$$
\pi\left(\left(a^{2}-b^{2} a\right) c\right)(1-c)=\pi\left(a^{2}-b^{2} a\right)\left(c-c^{2}\right)=-2 a^{2} c^{2} .
$$

Let $h_{4}=\left(a^{2}-b^{2} a\right) c b(1-a) c^{-p} a^{-2} h_{3}$. Then $\pi\left(h_{4}\right) 1=-2 a^{2} c^{2}$ and $\pi\left(h_{4}\right) x=0$ for all $x \in X \backslash\{1\}$. Thus, if we take $h=-\frac{1}{2}\left(a^{2} c^{2}\right)^{-1} h_{4}$, we get $\pi(h) 1=1$ and $\pi(h) x=0$ for all $x \in X \backslash\{1\}$.

Corollary 4.5. The representation $\pi$ is irreducible.

Proof. Follow the proof of Corollary 2.9.

\section{Identification method - second case}

Assume that there exist $a \in A$ and $b \in B$ with finite periods $p_{a}>2$ and $p_{b}>2$, respectively. Construct $L$ and define $\pi$ as in Section 4 . Then, arguing as before, we have that $\pi$ is a $*$-representation of $\mathbb{C}[G]$.

Here we define

$$
\begin{aligned}
V_{A}^{0} & =\left\{a^{s} u: 2<s<p_{a}, u \in U_{B}\right\}, \\
V_{B}^{0} & =\left\{b^{s} a u: 2<s<p_{b}, u \in U_{B}\right\}, \\
V_{A}^{\infty} & =\left\{a^{\prime} u: a^{\prime} \in A \backslash\left\{a^{s}: s \in \mathbb{Z}\right\}, u \in U_{B}\right\}, \\
V_{B}^{\infty} & =\left\{b^{\prime} u: b^{\prime} \in B \backslash\left\{b^{s}: s \in \mathbb{Z}\right\}, u \in U_{A}\right\},
\end{aligned}
$$

so that, as in Section 4, $V_{A} \backslash V_{A B}=V_{A}^{0} \cup V_{A}^{\infty}$ and $V_{B} \backslash V_{B A}=V_{B}^{0} \cup V_{B}^{\infty}$. Note that Lemma 4.2 holds in the present case.

Proposition 5.1. The representation $\pi$ is faithful.

Proof. Follow the proof of Proposition 2.3.

Lemma 5.2. (i) Let $w \in V_{A}^{0} \cup V_{A B}$. Then $\pi\left(c^{n}\right) w \in \pm U_{B}$ for all sufficiently large $n$.

(ii) Let $w \in U_{A} \cup V_{B}^{0}$. Then $\pi\left(c^{2 p_{b}-4}\right) w=w$.

Proof. (i) Follow the proof of Lemma 4.3 (ii) in the finite period case, where the definitions of $V_{A}^{0}$ and $V_{A}^{\infty}$ agree with those in this section. 
(ii) First, consider $w=a u \in U_{A}$ where $u \in U_{B}$. Then $\pi(a) w=a^{2} u \equiv \pm b^{2} a u$, and so $\pi(c) w= \pm \pi(b) b^{2} a u= \pm b^{3} a u$, and $b^{3} a u \in V_{B}^{0}$ if $p_{b}>3$. If $2<s<p_{b}$ then $\pi(c) b^{s} a u=$ $\pi(b) \pi(a) b^{s} a u=\pi(b) b^{s} a u=b^{s+1} a u$. It follows that $\pi\left(c^{p_{b}-2}\right) w= \pm b^{p_{b}} a u= \pm a u= \pm w$, and this also holds if $p_{b}=3$. Hence $\pi\left(c^{2 p_{b}-4}\right) w=w$.

Secondly, if $w \in V_{B}^{0}$ so that $w=b^{s} a u$ where $2<s<p_{b}$ and $u \in U_{B}$, then as above, $\pi\left(c^{p_{b}-s}\right) w=b^{p_{b}} a u=a u \in U_{A}$. So $\pi\left(c^{2 p_{b}-4}\right) w=\pi\left(c^{2 p_{b}-4}\right) \pi\left(c^{s-p_{b}}\right) a u=\pi\left(c^{s-p_{b}}\right) \pi\left(c^{2 p_{b}-4}\right) a u=$ $\pi\left(c^{s-p_{b}}\right) a u=w$.

Proposition 5.3. Let $X$ be a finite subset of $W$ and let $x_{0} \in X$. Then there exists $h \in \mathbb{C}[G]$ such that $\pi(h) x_{0}=1$ and $\pi(h) x=0$ for all $x \in X \backslash\left\{x_{0}\right\}$.

Proof. Without loss, take $x_{0}=1$. By Lemma 5.2, for all sufficiently large $p \in \mathbb{N}$,

$$
X_{1}=\pi\left(c^{p}\right) X \subseteq \pm\left(U_{A} \cup U_{B} \cup V_{B}^{0} \cup V_{A}^{\infty} \cup V_{B}^{\infty}\right)
$$

If we replace $p$ by $p+k\left(2 p_{b}-4\right)$ where $k \in \mathbb{N}$, then each element of $\operatorname{supp}\left(X_{1}\right) \cap\left(U_{A} \cup V_{B}^{0}\right)$ is unaltered while the elements of $\operatorname{supp}\left(X_{1}\right) \cap\left(U_{B} \cup V_{A}^{\infty} \cup V_{B}^{\infty}\right)$ remain in $U_{B} \cup V_{A}^{\infty} \cup V_{B}^{\infty}$. Since $a c^{p} \in U_{A}$, we may choose $p$ such that $a c^{p} \notin \operatorname{supp}\left(X_{1}\right)$. Then

$$
X_{2}=\pi(1-a) X_{1} \subseteq \operatorname{lin}\left(U_{A} \cup U_{B} \cup V_{A}\right) \text {, }
$$

since $\pi(1-a) w=0$ if $w \in V_{B}^{0} \cup V_{B}^{\infty}$, and $\pi(a) w \in V_{A B} \subseteq V_{A}$ if $w \in U_{A}$. By Lemma 5.2, we may choose $q$ to be a sufficiently large multiple of $2 p_{b}-4$ that

$$
X_{3}=\pi\left(c^{q}\right) X_{2} \subseteq \operatorname{lin}\left(U_{A} \cup\left(U_{B} \backslash S\right) \cup V_{A}^{\infty}\right)
$$

since $U_{B}$ is mapped into $U_{B} \backslash S$ and each element of $U_{A}$ is mapped to itself. Then

$$
X_{4}=\pi(b) X_{3} \subseteq \operatorname{lin}\left(U_{B} \cup V_{A}\right)
$$

since $\pi(b)\left(U_{B} \backslash S\right) \subseteq \pm V_{A B}$. By Lemma 5.2, we may choose $r \in \mathbb{N}$ such that

$$
X_{5}=\pi\left(c^{\prime}\right) X_{4} \subseteq \operatorname{lin}\left(\left(U_{B} \backslash S\right) \cup V_{A}^{\infty}\right),
$$

and then

$$
X_{6}=\pi(1-b) X_{5} \subseteq \operatorname{lin}\left(U_{B} \cup V_{A B}\right)
$$

since, again here, $\pi(b)\left(U_{B} \backslash S\right) \subseteq \pm V_{A B}$. For all sufficiently large $s \in \mathbb{N}$,

$$
X_{7}=\pi\left(c^{s}\right) X_{6} \subseteq \operatorname{lin}\left(U_{B}\right)
$$

Thus $X_{7}=\pi\left(h_{1}\right) X$ where

$$
h_{1}=c^{s}(1-b) c^{\prime} b c^{q}(1-a) c^{p}
$$


Let $u=c^{p+r+s+1}$ and suppose that $u \in \operatorname{supp}\left(X_{7}\right)$. This arises only from $\pi\left(c^{-s}\right) u=$ $c^{p+r+1} \in \operatorname{supp}\left(X_{6}\right)$ which, in turn, can come from either $c^{p+r+1}$ or $\pi\left(b^{-1}\right) c^{p+r+1}$ equalling $a c^{p+r}$ in $\operatorname{supp}\left(X_{5}\right)$. Since $\operatorname{supp}\left(X_{5}\right) \cap U_{A}=\emptyset$, only $c^{p+r+1} \in \operatorname{supp}\left(X_{5}\right)$ is possible. Then this is the image only of $\pi\left(c^{-r}\right) c^{p+r+1}=c^{p+1}$ in $\operatorname{supp}\left(X_{4}\right)$, then of $\pi\left(b^{-1}\right) c^{p+1}=a c^{p}$ in $\operatorname{supp}\left(X_{3}\right)$, and so of $\pi\left(c^{-q}\right) a c^{p}=a c^{p}$ in $\operatorname{supp}\left(X_{2}\right)$, since $\pi\left(c^{q}\right)$ fixes the elements of $U_{A}$. Now $a c^{p} \in \operatorname{supp}\left(X_{2}\right)$ comes from $a c^{p}$ or $\pi\left(a^{-1}\right) a c^{p}$ equalling $c^{p}$ in $\operatorname{supp}\left(X_{1}\right)$. Since $a c^{p} \notin \operatorname{supp}\left(X_{1}\right), c^{p}$ is the only precursor in $X_{1}$, and this arises only from $\pi\left(c^{-p}\right) c^{p}=1$ in $X$.

For $x \in W, \pi\left(h_{1}\right) x$ is a linear combination of four basis vectors, one of which is $\pi\left(h_{2}\right) x$ where $h_{2}=-c^{s+r} b c^{q} a c^{p}$. The above shows that if $x \in X \backslash\{1\}$ then $u \notin$ $\operatorname{supp}\left(\left\{\pi\left(h_{1}\right) x\right\}\right)$ while $\pi\left(h_{2}\right) 1=-u$, as may be easily verified, and the other vectors in $\pi\left(h_{1}\right) l$ involve elements of $U_{B} \backslash\{u\}$.

As in Proposition 2.8, choose $s$ sufficiently large that $\operatorname{supp}\left(X_{7}\right) \cap U_{B}^{*}=\{u\}$. Then $\pi\left(\left(a^{2}-b^{2} a\right) h_{1}\right) x=0$ if $x \in X \backslash\{1\}$, since then $\operatorname{supp}\left(\left\{\pi\left(h_{1}\right) x\right\}\right) \subseteq U_{B} \backslash U_{B}^{*}$, while $\pi\left(\left(a^{2}-\right.\right.$ $\left.\left.b^{2} a\right) h_{1}\right) 1=-\pi\left(a^{2}-b^{2} a\right) u=-2 a^{2} u$ by (4.1). Thus, taking

$$
h=-\frac{1}{2}\left(a^{2} u\right)^{-1}\left(a^{2}-b^{2} a\right) h_{1}
$$

gives $\pi(h) 1=1$ and $\pi(h) x=0$ for all $x \in X \backslash\{1\}$.

Corollary 5.4. The representation $\pi$ is irreducible.

Proof. Follow the proof of Corollary 2.9.

\section{Extending to $\ell^{1}(G)$}

Here we consider the algebra $\ell^{\prime}(G)$ which consists of the sums $\sum_{1}^{\infty} \alpha_{i} g_{i}\left(\alpha_{i} \in \mathbb{C}\right.$, $\left.g_{i} \in G, \sum_{1}^{\infty}\left|\alpha_{i}\right|<\infty\right)$. The four cases for $G$ dealt with in Sections 2 to 5 still apply but, for the most part, they can be treated together. The construction of $L$ and the definition of $\pi$ are the same as in these earlier sections except that $L$ now consists of the sums of the form $x=\sum_{1}^{\infty} \alpha_{i} w_{i}\left(\alpha_{i} \in \mathbb{C}, w_{i} \in W, \sum_{1}^{\infty}\left|\alpha_{i}\right|<\infty\right)$ with $\|x\|=\sum_{1}^{\infty}\left|\alpha_{i}\right|$ when $w_{i}$ are distinct.

Since, for $d \in A \cup B, \pi(d)$ acts on $W$ as a permutation followed by multiplication by \pm 1 , it follows that

$$
\|\pi(g) w\|=1, \quad\|\pi(g) x\|=\|x\| \quad(g \in G, w \in W, x \in L) .
$$

Then arguing as before, we have that $\pi$ is a $*$-representation of $\ell^{1}(G)$.

Proposition 6.1. The representation $\pi$ is faithful.

Proof. Let $f=\sum_{1}^{\infty} \alpha_{i} g_{i} \in \ell^{\prime}(G)$ with $x_{i} \in \mathbb{C}, \alpha_{1} \neq 0$ and $g_{i}$ distinct in $G$. Choose $n \in \mathbb{N}$ such that $\sum_{n+1}^{\infty}\left|\alpha_{i}\right|<\left|\alpha_{1}\right|$. Let $w$ be as in the proof of Proposition 2.3 with corresponding modifications for the other cases. Then 


$$
\left\|\sum_{n+1}^{\infty} \alpha_{i} \pi\left(g_{i}\right) w\right\| \leq \sum_{n+1}^{\infty}\left|\alpha_{i}\right|\left\|\pi\left(g_{i}\right) w\right\| \leq \sum_{n+1}^{\infty}\left|\alpha_{i}\right|<\left|\alpha_{1}\right|
$$

and, in view of Note 2.4 (1),

$$
\left\|\sum_{1}^{n} \alpha_{i} \pi\left(g_{i}\right) w\right\|=\left\|\sum_{1}^{n} \alpha_{i} g_{i} w\right\|=\sum_{1}^{n}\left|\alpha_{i}\right| \geq\left|\alpha_{1}\right| .
$$

Hence

$$
\|\pi(f) w\| \geq\left\|\sum_{1}^{n} \alpha_{i} \pi\left(g_{i}\right) w\right\|-\left\|\sum_{n+1}^{\infty} \alpha_{i} \pi\left(g_{i}\right) w\right\|>\left|\alpha_{1}\right|-\left|\alpha_{1}\right|=0 .
$$

Thus $\pi(f) \neq 0$.

The elements $h$ defined in the proofs of Propositions 2.8, 3.4, 4.4 and 5.3, when regarded as elements of $\ell^{\prime}(G)$, have the property that

$$
\|\pi(h) w\| \leq 8 \quad(w \in W)
$$

[In some cases, 8 can be replaced by a smaller integer.] For example, in the case of Proposition 4.4 , we have

$$
\begin{aligned}
h & =-\frac{1}{2}\left(a^{2} c^{2}\right)^{-1}\left(a^{2}-b^{2} a\right) c b(1-a) c^{-p} a^{-2}(1-a)(1-b) \frac{1}{2}\left(a^{2}-b^{2} a\right) c^{p} \\
& =\frac{1}{4}\left(\alpha_{1} g_{1}+\cdots+\alpha_{32} g_{32}\right),
\end{aligned}
$$

where $\alpha_{i}= \pm 1 \in \mathbb{C}$ and $g_{i} \in G$. Hence, using (6.1), for $w \in W$,

$$
\|\pi(h) w\| \leq \frac{1}{4}\left(\left\|\pi\left(g_{1}\right) w\right\|+\cdots+\left\|\pi\left(g_{32}\right) w\right\|\right) \leq 8 .
$$

The assumption in the proof of Proposition 4.4 that $x_{0}=1$ does not affect this argument.

Proposition 6.2. The representation $\pi$ is irreducible.

Proof. Let $x=\sum_{1}^{\infty} \alpha_{i} w_{i} \in L$ with $\alpha_{i} \in \mathbb{C}, \alpha_{1} \neq 0$ and $w_{i}$ distinct in $W$. Let $\varepsilon>0$. Choose $n \in \mathbb{N}$ such that $x=\sum_{n+1}^{\infty}\left|\alpha_{i}\right|<\varepsilon\left|\alpha_{1}\right| / 8$. Let $h$ be as in the proof of Corollary 2.9 with corresponding modifications for the other cases. Then

$$
\pi(h) x=\sum_{1}^{n} \alpha_{i} \pi(h) w_{i}+\sum_{n+1}^{\infty} \alpha_{i} \pi(h) w_{i}=\alpha_{1} 1+\sum_{n+1}^{\infty} \alpha_{i} \pi(h) w_{i} .
$$


Hence, using (6.2),

$$
\left\|\frac{1}{\alpha_{1}} \pi(h) x-1\right\|=\left\|\frac{1}{\alpha_{1}} \sum_{n+1}^{\infty} \alpha_{i} \pi(h) w_{i}\right\| \leq \frac{1}{\left|\alpha_{1}\right|} \sum_{n+1}^{\infty}\left|\alpha_{i}\right|\left\|\pi(h) w_{i}\right\| \leq \frac{8}{\left|\alpha_{1}\right|} \sum_{n+1}^{\infty}\left|\alpha_{i}\right|<\varepsilon .
$$

Since $\pi(w) 1=w$ for all $w \in W$, it follows that 1 in $L$ is cyclic. This together with the above gives $x$ topologically cyclic. Hence, since $L$ is complete, every non-zero vector in $L$ is cyclic (see, for example, McGregor [4, proof of Theorem 5]) and $\pi$ is irreducible.

\section{Concluding remarks}

(1) Combining the results of Sections 2 to 6 , we have constructed faithful, irreducible *-representations of $\mathbb{C}[G]$ and $\ell^{1}(G)$ for all cases of $G$ the free product of groups $A$ and $B$ with $|A| \geq 3$ and $|B| \geq 2$. The cases not dealt with explicitly all have $|B| \geq 3$ and are covered by interchanging the roles of $A$ and $B$.

(2) The constructions and conclusions obtained for $\mathbb{C}[G]$ and $\ell^{\prime}(G)$ hold for $\mathbb{R}[G]$ and the corresponding $\ell^{1}$-algebra over $\mathbb{R}$. They also hold, apart from the $*$-condition, for $\mathbb{F}[G]$ where $\mathbb{F}$ is any field not of characteristic 2 .

(3) The character method can be used for some cases covered by the identification method. The essential requirement for the character method is the existence of a nontrivial homomorphism from $B$ to $\mathbb{C}$, and this does not require $d^{2}=1$ for all $d \in B$.

(4) In all cases, the space $L$ is a subspace of the Hilbert space $\ell^{2}(W)$ consisting of the sums of the form $\sum_{1}^{\infty} \alpha_{i} w_{i}\left(\alpha_{i} \in \mathbb{C}, w_{i} \in W, \sum_{1}^{\infty}\left|\alpha_{i}\right|^{2}<\infty\right)$.

\section{REFERENCES}

1. E. Formanek, Group rings of free products are primitive, J. Algebra 26 (1973), 508-511.

2. R. S. IR VING, Some primitive group rings, J. Algebra 56 (1979), 274-281.

3. R. S. IRVING, Some more primitive group rings, Israel J. Math. 37 (1980), 4, 331-350.

4. C. M. McGregor, A representation for $\ell^{\prime}(S)$, Bull. London Math. Soc. 8 (1976), 156-160.

5. C. M. MCGregor, On the primitivity of the group ring of a free group, Bull. London Math. Soc. 8 (1976), 294-298.

6. W. L. Paschke and N. Salinas, $C^{*}$-algebras associated with free products of groups, Pacific J. Math. 82 (1979), 1, 211-221.

Department of Mathematics

UNIVERSITY OF GLASGOW

UNIVERSITY GARDENS

GLASGOW G12 8QW

SCOTLAND 